\title{
Barred Extreme IRAS Galaxies
}

\author{
Wim van Driel \\ Nançay Radio Astronomy Observatory, Observatoire de Paris, France \\ Bert van den Broek \\ Astronomical Institute, University of Amsterdam, The Netherlands
}

\begin{abstract}
We studied a statistically complete sample of 57 southern socalled extreme IRAS galaxies, i.e., objects with a high far-infrared/blue luminosity ratio, $\mathrm{L}_{F I R} / \mathrm{L}_{B}>3$, using optical (imaging and spectra), radio continuum, and $\mathrm{CO}(1-0)$ line observations. The sample can be divided into three distinct categories: dwarfs (20\%), barred spirals $(35 \%)$, and interacting systems (35\%). The barred galaxies are generally morphologically undisturbed, isolated systems, with average star formation rates $\left(4 \mathrm{M}_{\odot} \mathrm{yr}^{-1}\right)$ and efficiencies $\left(\mathrm{L}_{F I R} / \mathrm{M}_{H_{2}}=16 \mathrm{~L}_{\odot} / \mathrm{M}_{\odot}\right)$ for galaxies in our sample. An enhanced massive star formation rate is the cause of the infrared brightness in $93 \%$ of all galaxies in the sample. The nuclear region is the most important star formation locus, generally unresolved at $1^{\prime \prime}$ resolution, i.e., less than $0.2-0.6 \mathrm{kpc}$ size $\left(\mathrm{H}_{0}=75 \mathrm{~km} \mathrm{~s}^{-1} \mathrm{Mpc}^{-1}\right)$, though $2 \mathrm{kpc}$ size in three cases. In about two-thirds of the extreme IRAS SB's, fainter, diffuse (2.5-10 kpc size) massive star formation is seen in the bar as well.
\end{abstract}

\section{The Sample - Extreme IRAS Galaxies}

Most studies of so-called IRAS galaxies have concentrated on the very rare far-infrared ultraluminous objects with $\mathrm{L}_{\mathrm{FIR}} \gtrsim 10^{12} \mathrm{~L}_{\odot}$ detected by the IRAS satellite. These are generally distant interacting or merging objects with active nuclei.

For our studies of galaxies with enhanced massive star formation we have instead used the far-infrared/blue luminosity $\left(\mathrm{L}_{\mathrm{FIR}} / \mathrm{L}_{\mathrm{B}}\right)$ ratio as a selection criterion, in order also to include galaxies with normal luminosities in our sample.

The $L_{F I R} / L_{B}$ ratio as a selection ratio is a suitable criterion to select actively star forming galaxies, since it compares the far-infrared luminosity produced in the star forming process to the blue luminosity of the old stellar population.

We studied a statistically complete sample of 57 so-called extreme IRAS galaxies, i.e., objects with relatively high blue-to-infrared luminosity ratios ( $>3$ in solar units), between declinations of $-20^{\circ}$ and $-40^{\circ}$, i.e. readily accessible from ESO in Chile, at optical and radio wavelengths. 


\section{Observations - a Multi-Wavelength Study}

We made a multi-wavelength (infrared, optical and radio) study of the galaxies in our sample in order to get a broad spectral view, allowing a better interpretation of the enhanced star formation process occurring in most of them.

Of all galaxies in our sample we obtained:

- Broad-band $B, V, R$, and $\mathrm{H} \alpha$ CCD images (van den Broek et al. 1991a)

- Jow-resolution optical spectra (van den Broek et al. 1991a)

- VLA 6-cm radio continuum maps (van Driel et al. 1991b)

- CO(1-0) radio line spectra (van Driel et al. 1991a)

Optical broad-band imaging allows a determination of the morphological types and the color distributions, $\mathrm{H} \alpha$ line imaging a look at the massive star formation regions (though obscured by dust), VLA radio continuum imaging allows a view of the same regions unimpeded by dust, and through $\mathrm{CO}(1-0)$ radio line observations we can estimate the molecular gas mass and the star formation efficiency.

Tully-Fisher Relation: We also studied near-infrared aperture photometry and the near-infrared Tully-Fisher relation of a similar sample of IRAS galaxies, the so-called IRAS Minisurvey, which we observed in the $21-\mathrm{cm}$ HI line at Arecibo (van den Broek et al. 1991b; van Driel et al. 1995).

\section{Observations - Results}

In about $93 \%$ of the extreme IRAS galaxies we studied, the infrared emission is enhanced through intensive massive star formation, seen by their optical H IItype or LINER spectra; the others are Seyfert 2 objects.

Our sample can be almost completely divided into three distinct categories of objects (van den Broek 1993) - in order of increasing star formation rate:

- Dwarfs (20\%)

- Barred spirals (35\%)

- Interacting systems (35\%)

Note that there are no normal non-barred spirals in our sample.

The dwarfs, the smallest systems, have the largest star forming regions of size 2-6 kpc $\left(\mathrm{H}_{0}=75 \mathrm{~km} \mathrm{~s}^{-1} \mathrm{Mpc}^{-1}\right)$, usually spread over a large fraction of their disk, while the interacting systems have the most compact star forming regions, which often remain unresolved at $1^{\prime \prime}$ resolution, usually located in their nuclei, with sizes ranging from less than one $\mathrm{kpc}$ to several $\mathrm{kpc}$.

\subsection{The Barred Extreme IRAS Galaxies}

Star Formation - Rates and Efficiences: Practically all barred extreme IRAS galaxies have normal optical morphologies showing a range of ring subtypes, and appear to be isolated systems. They have intermediate star formation rates (average $4 \mathrm{M}_{\odot} \mathrm{yr}^{-1}$ ) and star formation efficiencies (average $\mathrm{L}_{F I R} / \mathrm{M}_{H_{2}}=16 \mathrm{~L}_{\odot}$ $\mathrm{M}_{\odot}^{-1}$ ) for objects in our sample. 
Star Formation Regions: In all barred systems the nuclear region is the most important locus of massive star formation, generally unresolved and less than $0.2-0.6 \mathrm{kpc}$ size but $2 \mathrm{kpc}$ size in three cases, though in about two-thirds of them lower-level, more diffuse $(2.5-10 \mathrm{kpc}$ size) star formation regions are seen in the bar as well. In only two cases do we find evidence for a resolved circumnuclear zone of massive star formation. A few of the most luminous barred spirals in our sample have LINER spectra, which may point to a mild form of nuclear activity.

Starburst Model: Using a simple exponential-decay starburst model, we analyzed the available multi-wavelength data of our extreme IRAS galaxies sample and the similar IRAS Minisurvey sample. We thus estimated an average exponential decay time of order 10 million years for the starbursts occurring in the galaxies (van den Broek et al. 1991b; van den Broek 1992; van Driel et al. 1995).

Star Formation in Barred Extreme IRAS Galaxies: In isolated gas-rich disk galaxies the presence of a non-axisymmetric bar potential is obviously a necessary condition to stir up the disk gas and to enhance the formation of massive stars. Apparently, the density waves causing spiral arms in normal non-barred spiral disks are not enough to really raise the star formation activity in a galaxy; we therefore do not find any of them among the extreme IRAS galaxies. In strongly interacting sytems obviously strong shocks are occurring which can enhance the star formation even further. The wide-spread star formation seen in dwarf galaxies may well be a stochastic process.

\section{References}

van den Broek, A. C. 1992, A\&A, 261, L1

van den Broek, A. C. 1993, A\&A, 269, 96

van den Broek, A. C., van Driel, W., de Jong, T., Lub, J., de Grijp, M. H. K., \& Goudfrooij, P. 1991a, A\&AS, 91, 61

van den Broek, A. C., de Jong, T., \& Brink, K. 1991b, A\&A, 246, 313

van Driel, W. \& van den Broek, A. C. 1991a, A\&A, 251, 431

van Driel, W., van den Broek, A. C., \& de Jong, T. 1991b, A\&AS, 90, 55

van Driel, W., van den Broek, A. C., \& Baan, W. 1995, ApJ, in press 